Supporting Information

\title{
Intrinsic Descriptors for Coordination Environment and Synergistic Effects of Metal and Environment in Single-Atom-Catalyzed Carbon Dioxide Electroreduction
}

Hao Yuan and Zhenyu Li*

Hefei National Laboratory for Physical Sciences at the Microscale, University of Science and Technology of China, Hefei, Anhui 230026, China

Email: zyli@ustc.edu.cn 
Table S1. Energy difference between opposite $\left(\mathrm{Co} @ \mathrm{X}_{n} \mathrm{Y}_{4-\mathrm{n}-\mathrm{O}}\right)$ and neighboring $\left(\mathrm{Co} @ \mathrm{X}_{\mathrm{n}} \mathrm{Y}_{4-\mathrm{n}-\mathrm{n}}\right)$ coordination sites of $\mathrm{X}$ or $\mathrm{Y}$ atoms in $\mathrm{Co} @ \mathrm{X}_{\mathrm{n}} \mathrm{Y}_{4-\mathrm{n}}$. The energy of Co@ $\mathrm{X}_{\mathrm{n}} \mathrm{Y}_{4-\mathrm{n}-\mathrm{n}}$ is site to zero.

\begin{tabular}{|c|c|}
\hline $\mathrm{Co} @ \mathrm{C}_{2} \mathrm{~N}_{2}-\mathrm{n}$ & 0 \\
\hline $\mathrm{Co} @ \mathrm{C}_{2} \mathrm{~N}_{2}-\mathrm{o}$ & 0.16 \\
\hline $\mathrm{Co} @ \mathrm{C}_{2} \mathrm{P}_{2}-\mathrm{n}$ & 0 \\
\hline $\mathrm{Co} @ \mathrm{C}_{2} \mathrm{P}_{2-\mathrm{o}}$ & 0.84 \\
\hline $\mathrm{Co} @ \mathrm{C}_{2} \mathrm{~S}_{2}-\mathrm{n}$ & 0 \\
\hline $\mathrm{Co} @ \mathrm{C}_{2} \mathrm{~S}_{2}-\mathrm{o}$ & 0.15 \\
\hline $\mathrm{Co} @ \mathrm{~N}_{2} \mathrm{P}_{2}-\mathrm{n}$ & 0 \\
\hline $\mathrm{Co} @ \mathrm{~N}_{2} \mathrm{P}_{2}-\mathrm{o}$ & 0.44 \\
\hline $\mathrm{Co} @ \mathrm{~N}_{2} \mathrm{~S}_{2}-\mathrm{n}$ & 0 \\
\hline $\mathrm{Co} @ \mathrm{~N}_{2} \mathrm{~S}_{2}-\mathrm{o}$ & 0.47 \\
\hline $\mathrm{Co} @ \mathrm{P}_{2} \mathrm{~S}_{2}-\mathrm{n}$ & 0 \\
\hline $\mathrm{Co} @ \mathrm{P}_{2} \mathrm{~S}_{2}-\mathrm{o}$ & 0.05 \\
\hline
\end{tabular}

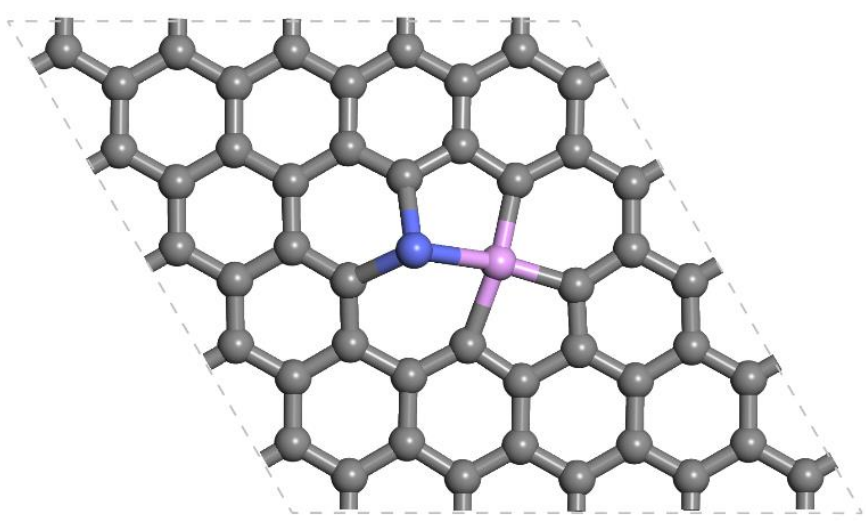

Figure S1. The geometric structure of $\mathrm{Co} @ \mathrm{C}_{3} \mathrm{P}_{1}$. The gray, light blue, and pink represent $\mathrm{C}, \mathrm{Co}$, and $\mathrm{P}$ atoms, respectively. Co atom has a coordination number 3 and $\mathrm{P}$ atom has a coordination number 4 . 
Table S2. d-band center of spin up and spin down orbitals of $\mathrm{Co} @ \mathrm{C}_{4}, \mathrm{Co} @ \mathrm{~N}_{4}, \mathrm{Co} @ \mathrm{P}_{4}$ and $\mathrm{Co@S} \mathrm{S}_{4}$ catalysts.

\begin{tabular}{|c|c|c|}
\hline & up & Down \\
\hline $\mathrm{Co@} \mathrm{C}_{4}$ & -1.69 & -0.70 \\
\hline $\mathrm{Co@N} \mathrm{N}_{4}$ & -1.47 & -0.65 \\
\hline $\mathrm{Co@} \mathrm{P}_{4}$ & -1.31 & -1.04 \\
\hline $\mathrm{Co@} \mathrm{S}_{4}$ & -0.86 & -0.86 \\
\hline
\end{tabular}

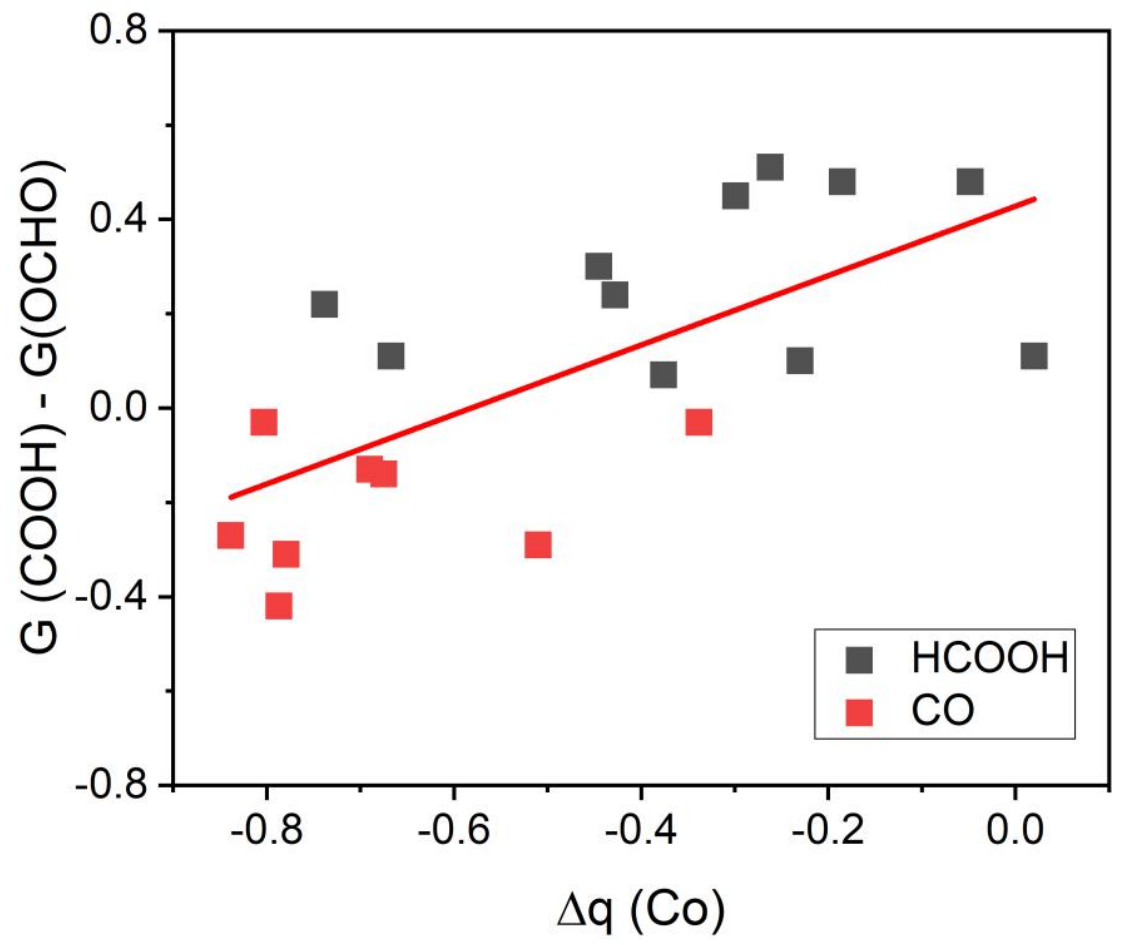

Figure S2. The scaling relationship between the lost electrons of Co and preferred reaction path $(\mathrm{G}(\mathrm{COOH})-\mathrm{G}(\mathrm{OCHO}))$. Red and black box represent reaction pathway to produce $\mathrm{CO}$ and $\mathrm{HCOOH}$, respectively. 

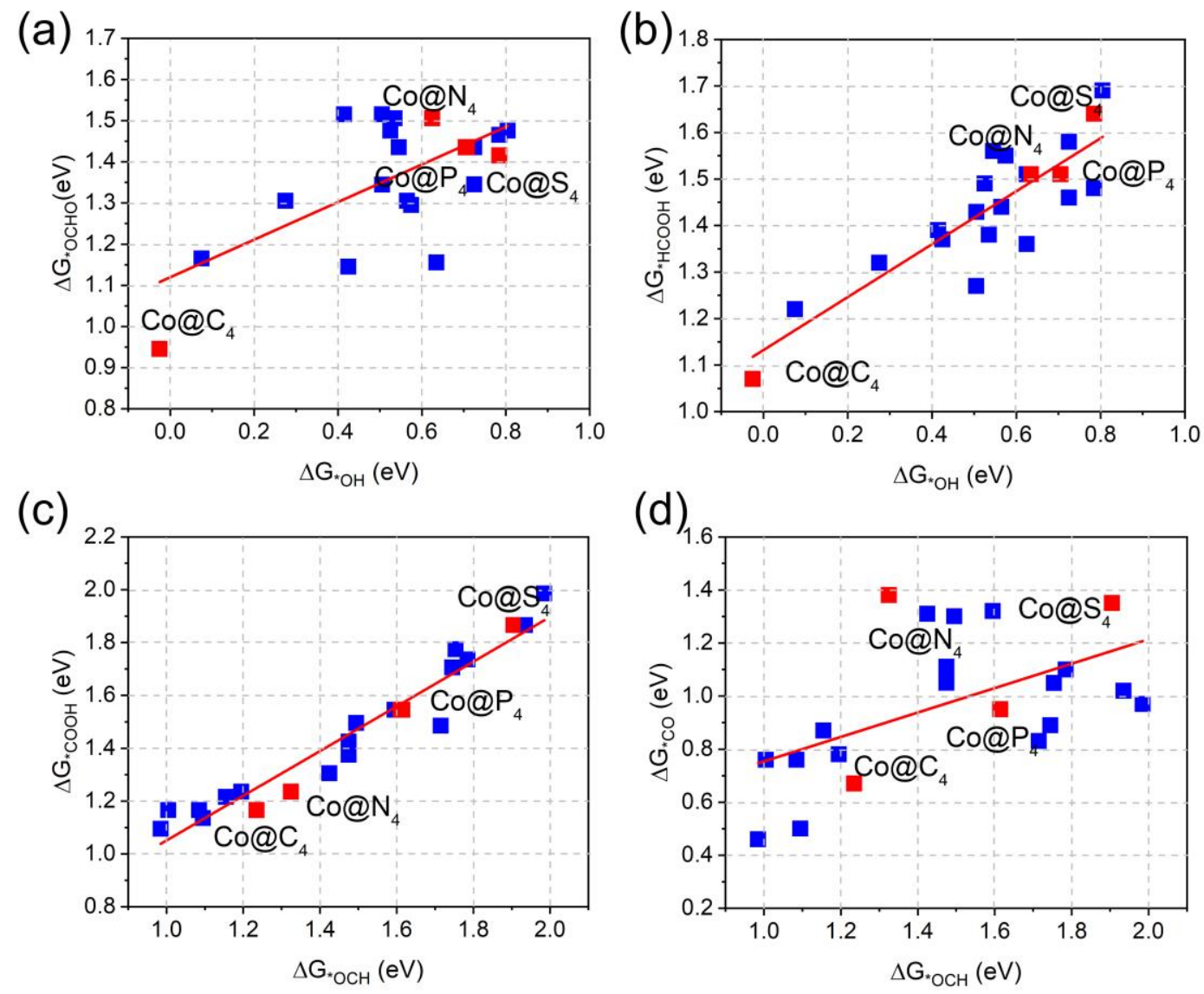

Figure S3. Scaling relationships between adsorption free energy of $* \mathrm{OCHO}$ and $\Delta \mathrm{G} * \mathrm{OH}$, between adsorption free energy of $* \mathrm{HCOOH}$ and $\Delta \mathrm{G} * \mathrm{OH}$, between adsorption free energy of ${ }^{*} \mathrm{COOH}$ and $\Delta \mathrm{G} * \mathrm{OCH}$, and between adsorption free energy of $* \mathrm{CO}$ and $\Delta \mathrm{G} * \mathrm{OCH}$. The catalysts of $\mathrm{Co} @ \mathrm{C}_{4}, \mathrm{Co} @ \mathrm{~N}_{4}, \mathrm{Co} @ \mathrm{P}_{4}$ and $\mathrm{Co} @ \mathrm{~S}_{4}$ are highlight in red.

Table S3. Linear correlations for Co@ $\mathrm{X}_{\mathrm{n}} \mathrm{Y}_{4-\mathrm{n}}(\mathrm{X}, \mathrm{Y}=\mathrm{C}, \mathrm{N}, \mathrm{P}, \mathrm{S})$

$\Delta \mathrm{G} * \mathrm{OCHO}=0.456 * \Delta \mathrm{G} * \mathrm{OH}+1.12$

$\Delta \mathrm{G} * \mathrm{HCOOH}=0.569 * \Delta \mathrm{G} * \mathrm{OH}+1.132$

$\Delta \mathrm{G} * \mathrm{COOH}=0.846 * \Delta \mathrm{G} * \mathrm{OCH}+0.205$

$\Delta \mathrm{G} * \mathrm{CO}=0.46 * \Delta \mathrm{G} * \mathrm{OCH}+0.294$ 
Table S4. Linear correlations for $\mathrm{Co} @ \mathrm{X}_{4}(\mathrm{X}=\mathrm{C}, \mathrm{N}, \mathrm{P}, \mathrm{S})$

$\Delta \mathrm{G} * \mathrm{OCHO}=0.655 * \Delta \mathrm{G} * \mathrm{OH}+0.983$

$\Delta \mathrm{G} * \mathrm{HCOOH}=0.667 * \Delta \mathrm{G} * \mathrm{OH}+1.084$

$\Delta \mathrm{G} * \mathrm{COOH}=1.056 * \Delta \mathrm{G} * \mathrm{OCH}-0.152$

$\Delta \mathrm{G} * \mathrm{CO}=0.542 * \Delta \mathrm{G} * \mathrm{OCH}+0.263$

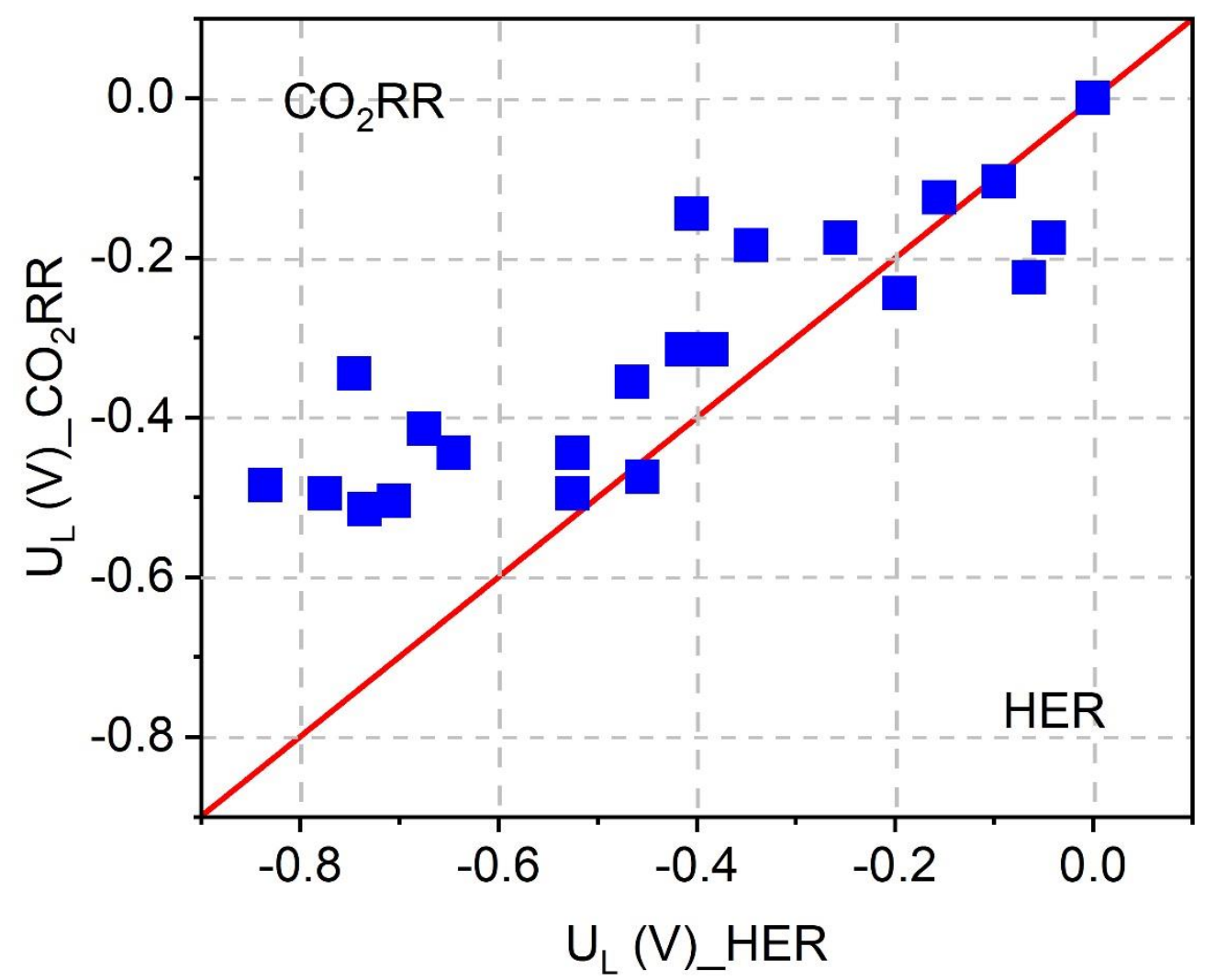

Figure S4. Limiting potential of hydrogen evolution reaction (HER) vs limiting potential of $\mathrm{CO}_{2} \mathrm{RR}$. 
Table S5. Input features for extra tree regression. These data are extracted from NIST chemistry webbook.

\begin{tabular}{llllllllr}
\hline coordination & EN & F_IE & S_IE & T_IE & \multicolumn{1}{c}{ EA } & p & CR & \multicolumn{1}{c}{ AN } \\
\hline $\mathrm{C}_{4}$ & 2.55 & 11.26 & 24.38 & 47.89 & 1.22 & 2.00 & 0.76 & 6.00 \\
$\mathrm{~N}_{4}$ & 3.04 & 14.53 & 29.60 & 47.45 & -0.07 & 3.00 & 0.71 & 7.00 \\
$\mathrm{P}_{4}$ & 2.19 & 10.49 & 19.77 & 30.20 & 0.72 & 3.00 & 1.07 & 15.00 \\
$\mathrm{~S}_{4}$ & 2.58 & 10.36 & 23.34 & 34.79 & 2.00 & 4.00 & 1.05 & 16.00 \\
$\mathrm{C}_{3} \mathrm{~N}_{1}$ & 2.67 & 12.08 & 25.69 & 47.78 & 0.90 & 2.25 & 0.75 & 6.25 \\
$\mathrm{C}_{3} \mathrm{~S}_{1}$ & 2.56 & 11.04 & 24.12 & 44.61 & 1.42 & 2.50 & 0.83 & 8.50 \\
$\mathrm{~N}_{3} \mathrm{C}_{1}$ & 2.92 & 13.72 & 28.30 & 47.56 & 0.25 & 2.75 & 0.72 & 6.75 \\
$\mathrm{~N}_{3} \mathrm{P}_{1}$ & 2.83 & 13.52 & 27.14 & 43.14 & 0.13 & 3.00 & 0.80 & 9.00 \\
$\mathrm{~N}_{3} \mathrm{~S}_{1}$ & 2.93 & 13.49 & 28.04 & 44.28 & 0.45 & 3.25 & 0.80 & 9.25 \\
$\mathrm{P}_{3} \mathrm{C}_{1}$ & 2.28 & 10.68 & 20.92 & 34.62 & 0.85 & 2.75 & 0.99 & 12.75 \\
$\mathrm{P}_{3} \mathrm{~N}_{1}$ & 2.40 & 11.50 & 22.23 & 34.51 & 0.52 & 3.00 & 0.98 & 13.00 \\
$\mathrm{P}_{3} \mathrm{~S}_{1}$ & 2.29 & 10.46 & 20.66 & 31.35 & 1.04 & 3.25 & 1.07 & 15.25 \\
$\mathrm{~S}_{3} \mathrm{C}_{1}$ & 2.57 & 10.59 & 23.60 & 38.06 & 1.81 & 3.50 & 0.98 & 13.50 \\
$\mathrm{~S}_{3} \mathrm{~N}_{1}$ & 2.70 & 11.40 & 24.90 & 37.95 & 1.48 & 3.75 & 0.97 & 13.75 \\
$\mathrm{~S}_{3} \mathrm{P}_{1}$ & 2.48 & 10.39 & 22.45 & 33.64 & 1.68 & 3.75 & 1.06 & 15.75 \\
$\mathrm{C}_{2} \mathrm{~N}_{2}$ & 2.80 & 12.90 & 26.99 & 47.67 & 0.58 & 2.50 & 0.74 & 6.50 \\
$\mathrm{C}_{2} \mathrm{P}_{2}$ & 2.37 & 10.87 & 22.08 & 39.05 & 0.97 & 2.50 & 0.92 & 10.50 \\
$\mathrm{C}_{2} \mathrm{~S}_{2}$ & 2.57 & 10.81 & 23.86 & 41.34 & 1.61 & 3.00 & 0.91 & 11.00 \\
$\mathrm{~N}_{2} \mathrm{P}_{2}$ & 2.62 & 12.51 & 24.69 & 38.83 & 0.33 & 3.00 & 0.89 & 11.00 \\
$\mathrm{~N}_{2} \mathrm{~S}_{2}$ & 2.81 & 12.45 & 26.47 & 41.12 & 0.97 & 3.50 & 0.88 & 11.50 \\
$\mathrm{P}_{2} \mathrm{~S}_{2}$ & 2.39 & 10.42 & 21.55 & 32.50 & 1.36 & 3.50 & 1.06 & 15.50
\end{tabular}

Average electronegativity (EN), average first ionization energy (F_IE), average second ionization energy (S_IE), average third ionization energy (T_IE) in $\mathrm{kj} / \mathrm{mol}$, average electron affinity (EA) in eV, average electrons in outer $p$-orbital (p), average covalent radius (CR) in $\AA$, average atomic number (AN).

(a)

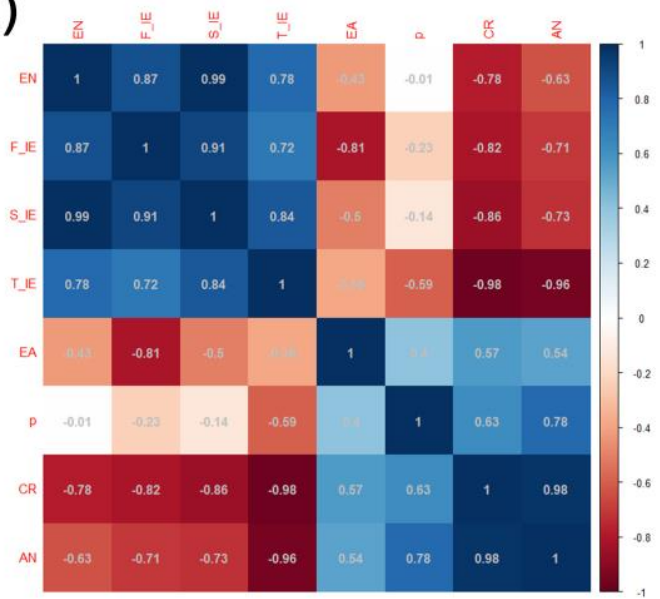

(b)

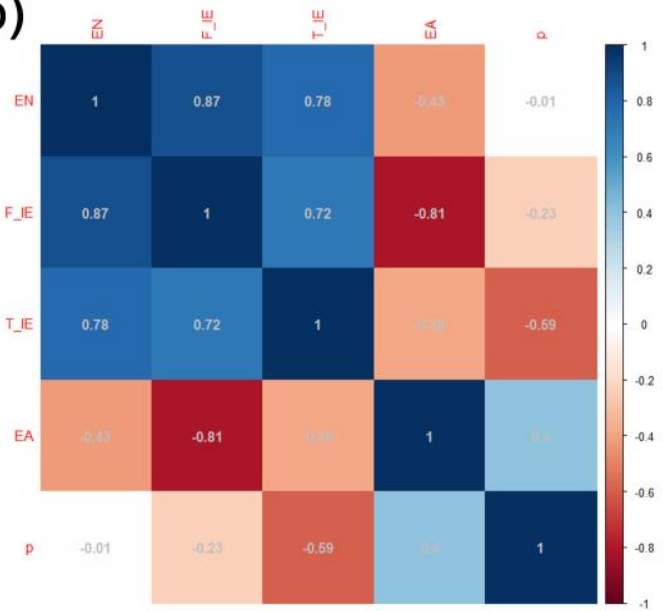

Figure S5. (a) Correlation map for eight features. There are high correlation between 
EN and S_IE (0.99), between T_IE and CR (-0.98), as well as between T_IE and AN (0.96). Therefore, S_IE, CR and AN are excluded. Five independent features are shown in (b). The correlation values are shown in color and values in the box.

(a)

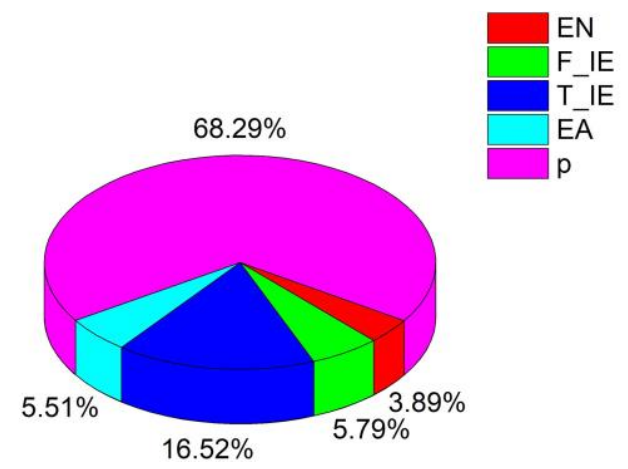

(b)

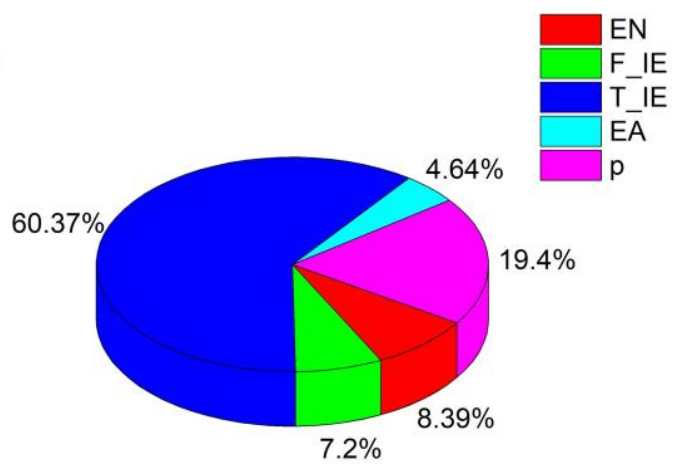

Figure S6. Feature importance extracted from the ETR model. (a) and (b) represent feature importance of the adsorption free energy of $* \mathrm{OH}$ and $* \mathrm{OCH}$, respectively.

(a)

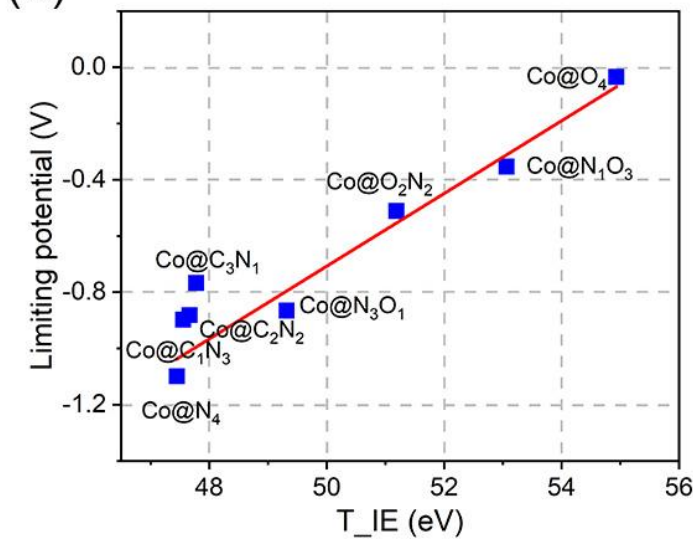

(b)

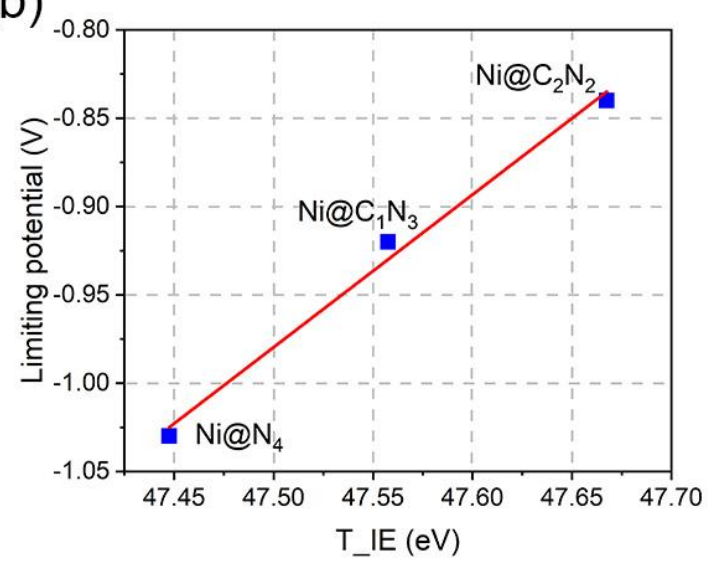

Figure S7. (a) Scaling relationships between descriptor T_IE and limiting potentials in

$\mathrm{Co} @ \mathrm{X}_{\mathrm{n}} \mathrm{Y}_{4-\mathrm{n}}(\mathrm{X}, \mathrm{Y}=\mathrm{C}, \mathrm{N}, \mathrm{O})^{1}$. (b) The linear correlation between descriptor T_IE and limiting potential in aqueous solution with an electrode potential of $0.65 \mathrm{~V}^{2}$. 


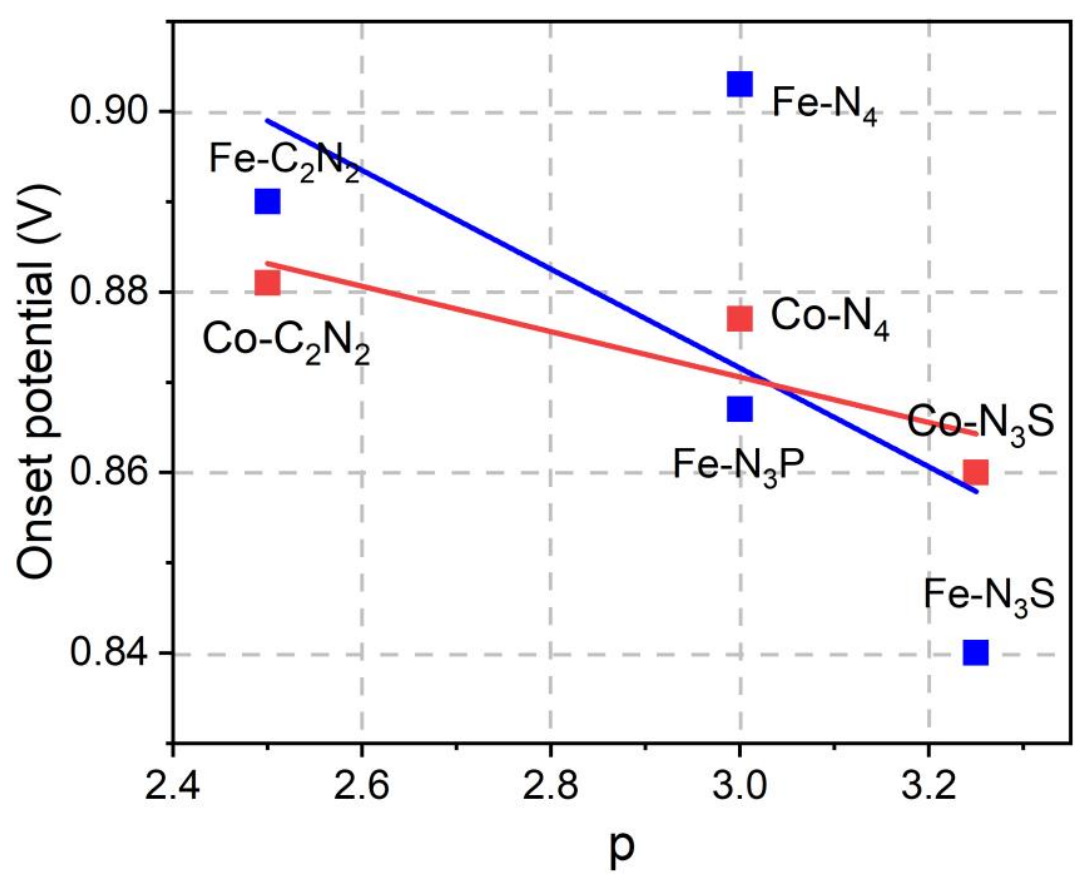

Figure S8. (a) Scaling relationships between average number of electrons in outer $p$ orbitals and onset potential for $\mathrm{ORR}^{3}$. The active Fe and Co centers are represented in blue and red box, respectively.
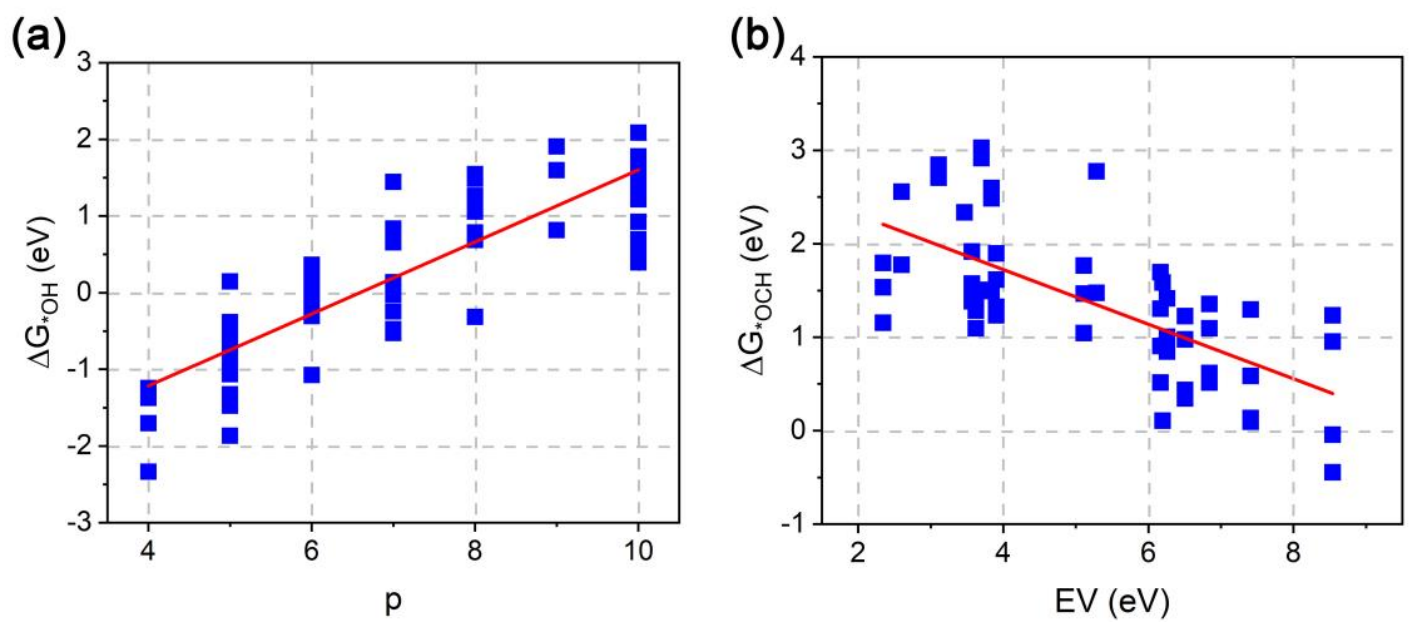

Figure S9. Scaling relationships between (a) adsorption free energy of $* \mathrm{OH}$ and descriptor $\mathrm{p}$, and (b) between adsorption free energy *OCH and descriptor EV.

Table S6. Liner correlations with or without the effect of coordination environment: 
${ }^{*} \mathrm{OH}: \quad \triangle \mathrm{G} * \mathrm{OH}=0.47 * \mathrm{~d}-3.09\left(\mathrm{R}^{2}=0.716\right)$

$$
\triangle \mathrm{G} * \mathrm{OH}=-29.25 * \Phi * \mathrm{OH}+3.37\left(\mathrm{R}^{2}=0.756\right)
$$

$* \mathrm{OCH}: \quad \triangle \mathrm{G} * \mathrm{OCH}=-0.29 * \mathrm{EV}+2.89\left(\mathrm{R}^{2}=0.435\right)$

$$
\triangle \mathrm{G} * \mathrm{OCH}=-0.004 * \Phi * \mathrm{OH}+2.2\left(\mathrm{R}^{2}=0.450\right)
$$

(a)

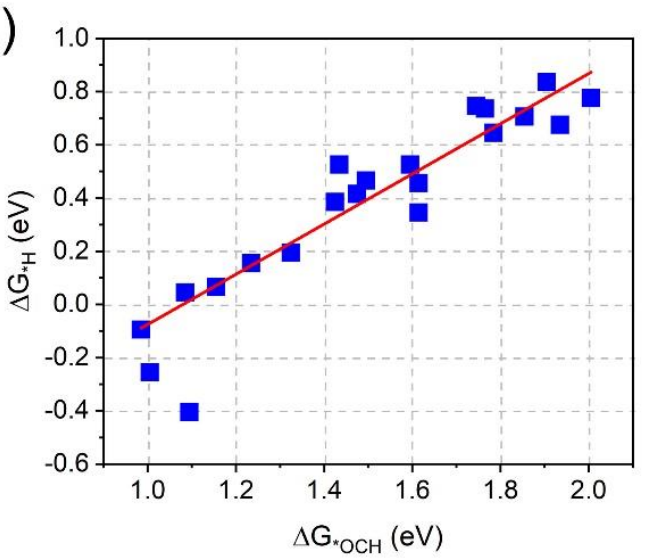

(b)

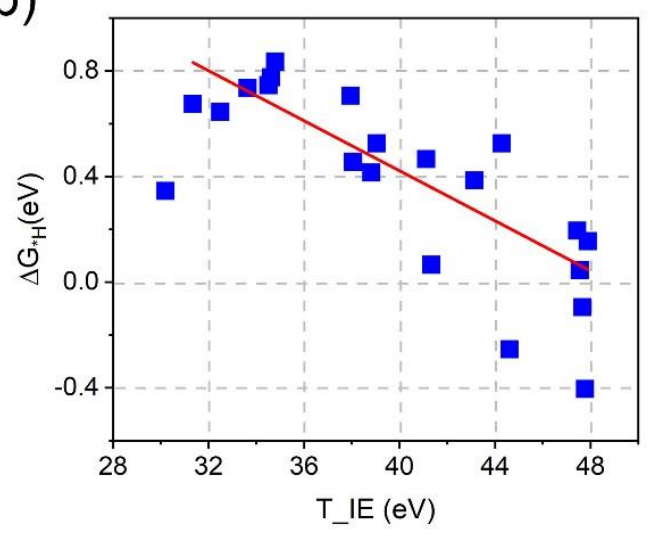

Figure S10. (a) The linear correlations between descriptor $\Delta \mathrm{G} * \mathrm{OCH}$ and $\Delta \mathrm{G} *_{\mathrm{H}}$. (b) The linear correlation between descriptor T_IE and $\Delta \mathrm{G} * \mathrm{H}$.

Table S7. Experimental SACs for ORR in Figure 4c.

\begin{tabular}{lccr}
\hline \multicolumn{1}{c}{ Catalysts } & $\begin{array}{c}\mathrm{E}_{1 / 2}(\mathrm{~V} \text { vs } \\
\mathrm{RHE})\end{array}$ & Solution & \multicolumn{1}{c}{ Reference } \\
\hline $\mathrm{Cu} @ \mathrm{~N}_{4}$ & 0.895 & $0.1 \mathrm{M} \mathrm{KOH}$ & $\begin{array}{r}\text { Nat Catal 2018, 1, } \\
781-786\end{array}$ \\
\hline $\mathrm{Pt} @ \mathrm{~N}_{4}$ & 0.887 & $0.1 \mathrm{M} \mathrm{KOH}$ & $\begin{array}{r}\text { ACS Catal 2018, 8, } \\
8450-8458\end{array}$ \\
& & & $\begin{array}{r}\text { Adv Funct Mater } \\
\mathrm{Co} @ \mathrm{~N}_{4}\end{array}$ \\
& 0.877 & $0.1 \mathrm{M} \mathrm{KOH}$ & $2018,28,1704638$. \\
\hline $\mathrm{Co} @ \mathrm{C}_{2} \mathrm{~N}_{2}$ & 0.881 & $0.1 \mathrm{M} \mathrm{KOH}$ & $\begin{array}{r}\text { Angew Chem Int } \\
\text { Edit 2016, 55, } \\
10800-10805\end{array}$ \\
& & & $\begin{array}{r}\mathrm{J} \text { Am Chem Soc } \\
2019,141,20118-\end{array}$ \\
\hline $\mathrm{Co} @ \mathrm{~N}_{3} \mathrm{~S}$ & 0.86 & $0.1 \mathrm{M} \mathrm{KOH}$ &
\end{tabular}




\begin{tabular}{lrrr}
\hline & & & 20126 \\
\hline $\mathrm{Fe} @ \mathrm{~N}_{3} \mathrm{P}$ & & & $\mathrm{J}$ Am Chem Soc \\
& 0.867 & $0.1 \mathrm{M} \mathrm{KOH}$ & $2020,142,2404-$ \\
& & 2412 \\
\hline $\mathrm{Fe} @ \mathrm{~N}_{3} \mathrm{~S}$ & 0.84 & $0.1 \mathrm{M} \mathrm{KOH}$ & $\begin{array}{r}\text { Small 2019, } 15, \\
1900307\end{array}$ \\
\hline $\mathrm{Fe} @ \mathrm{~N}_{4}$ & & & $\mathrm{~J}$ Mater Chem A \\
& 0.811 & $0.1 \mathrm{M} \mathrm{KOH}$ & $2019,7,26147-$ \\
& & & 26153 \\
\hline
\end{tabular}

Table S8. Experimental SACs for $\mathrm{CO}_{2} \mathrm{RR}$ in Figure 4d.

\begin{tabular}{|c|c|c|}
\hline Catalysts & $\mathrm{E}_{\text {onset }}$ (vs. RHE) & Reference \\
\hline $\mathrm{Ni} @ \mathrm{C}_{3} \mathrm{~N}$ & -0.31 & $\begin{array}{l}\text { Energy Environ. Sci. } \\
2018,11,893-903\end{array}$ \\
\hline $\mathrm{Cu} @ \mathrm{~N}_{4}$ & -0.16 & $\begin{array}{r}\text { Nat. Commun. } 2020, \\
11,2455\end{array}$ \\
\hline $\mathrm{Pd} @ \mathrm{~N}_{4}$ & -0.2 & $\begin{array}{l}\text { Adv. Funct. Mater. } \\
2020,30,2000407\end{array}$ \\
\hline $\mathrm{Ni@N_{4 }}$ & -0.4 & $\begin{array}{r}\text { J. Am. Chem. } \\
\text { Soc. } 2017,139,42, \\
14889-14892 \\
\end{array}$ \\
\hline $\mathrm{Fe} @ \mathrm{~N}_{4}$ & -0.31 & $\begin{array}{r}\text { ACS Catal. 2017, } 7 \\
1520-1525 \\
\end{array}$ \\
\hline $\mathrm{Co} @ \mathrm{~N}_{4}$ & -0.35 & $\begin{array}{r}\text { Appl. Catal., B 2019, } \\
240234-240\end{array}$ \\
\hline
\end{tabular}

\section{Reference}

1. Zhou, H.; Zou, X.; Wu, X.; Yang, X.; Li, J., Coordination Engineering in Cobalt-NitrogenFunctionalized Materials for $\mathrm{CO}_{2}$ Reduction. J. Phys. Chem. Lett. 2019, 10, 6551-6557.

2. Zhao, X.; Liu, Y. Unveiling the Active Structure of Single Nickel Atom Catalysis: Critical Roles of Charge Capacity and Hydrogen Bonding. J. Am. Chem. Soc. 2020, 142, 5773-5777.

3. Lu B, Liu Q, Chen S. Electrocatalysis of single-atom sites: impacts of atomic coordination. ACS Catal. 2020, 10, 7584-7618. 